The detection of $\mathrm{O}_{3}$ on Rhea and Dione is the first evidence that the accumulation of oxygen and ozone in icy surfaces exposed to ion irradiation may be a widespread process with significance in at least two areas outside the study of the Solar System. Molecular oxygen has been predicted to be abundant in the mantles of interstellar grains in dense clouds ${ }^{25}$ and production of $\mathrm{O}_{3}$ in these grains via irradiation by cosmic rays, stellar winds, or ultraviolet light may be an important factor to consider in models of grain chemistry ${ }^{11}$. Possibly of greater significance, plans to search for and identify Earth-like planets around other stars frequently cite the $9.8-\mu \mathrm{m}$ band of $\mathrm{O}_{3}$ as a means to identify planets with massive $\mathrm{O}_{2}$ atmospheres $^{26}$. A significant component of the Earth's atmosphere was probably derived from impacts of icy comets ${ }^{27}$. If the ices in these comets contain excess oxygen produced by irradiation as the comet accretes, the volatiles delivered to a planetary atmosphere in this way could contain as much as a few per cent $\mathrm{O}_{2}$ by mass (in excess of the oxygen contained in $\mathrm{H}_{2} \mathrm{O}$ ice). Because ozone is a very nonlinear tracer of molecular oxygen in a planetary atmosphere, an Earth-like atmosphere with a partial pressure of $\mathrm{O}_{2}$ of only $2 \mathrm{mbar}$, an amount that could conceivably be supplied by $\mathrm{O}_{2}$-saturated ices, would have a detectable column of $\mathrm{O}_{3}$ only one-quarter that in Earth's present atmosphere ${ }^{28}$ ( 2 mbar is $2 \%$ of Earth's sea-level pressure or $\sim 10 \%$ of the $\mathrm{O}_{2}$ currently in the atmosphere). The identification of oxygen-containing atmospheres on extrasolar planets through detection of $\mathrm{O}_{3}$ may not, therefore, be an unambiguous way of identifying planets with Earth-like biology.

Received 27 March; accepted 13 May 1997

1. Clark, R. N. Ganymede, Europa, Callisto, and Saturn's rings: Compositional analysis from reflectance spectroscopy. Icarus 44, 388-409 (1980).

. Clark, R. N., Brown, R. H., Owensby, P. D. \& Steele, A. Saturn's satellites: Near-infrared spectrophotometry $(0.65-2.5 \mu \mathrm{m})$ of the leading and trailing sides and compositional implications. Icarus 58, 265-281 (1984)

. Johnson, R. E. Energetic Charged Particle Interactions with Atmospheres and Surfaces (Springer, Berlin, 1990).

4. Hall, D. T., Strobel, D. F., Feldman, P. D., McGrath, M. A. \& Weaver, H. A. Detection of an oxygen atmosphere on Jupiter's moon Europa. Nature 373, 677-679 (1995).

5. Barth, C. A. et al. Galileo ultraviolet observations of atomic hydrogen in the atmosphere of Ganymede. Geophys. Res. Lett. (in the press).

6. Spencer, J. R., Calvin, W. M. \& Person, M. J. Charge-coupled device spectra of the galilean satellites: Molecular oxygen on Ganymede. J. Geophys. Res. 100, 19049-19056 (1995).

7. Noll, K. S., Johnson, R. E., Lane, A. L., Domingue, D. \& Weaver, H. A. Detection of ozone on Ganymede. Science 273, 341-343 (1996).

8. Woods, T. N., Rottman, G. J. \& Ucker, G. J. Solar-stellar irradiance comparison experiment 1: 2. Instrument calibrations. J. Geophys. Res. 98, 10679-10694 (1993).

9. Buratti, B. \& Veverka, J. Voyager photometry of Rhea, Dione, Tethys, Enceladus, and Mimas. Icarus 58, 254-264 (1984).

10. Morrison, D., Jones, T. J., Cruikshank, D. P. \& Murphy, R. E. The two faces of Iapetus. Icarus 24, 157 171 (1975).

11. Elsila, J., Allamandola, L. J. \& Sandford, S. A. The $2140 \mathrm{~cm}^{-1}$ (4.673 microns) solid CO band: The case for interstellar ice analogs. Astrophys. J. (in the press).

12. Matich, A. J., Bakker, M. G., Lennon, D., Quickenden, T. I. \& Freeman, C. G. $\mathrm{O}_{2}$ luminescence from UV-excited $\mathrm{H}_{2} \mathrm{O}$ and $\mathrm{D}_{2} \mathrm{O}$ ices. J. Phys. Chem. 97, 10539-10553 (1993).

13. Johnson, R. E. \& Jesser, W. A. $\mathrm{O}_{2} / \mathrm{O}_{3}$ microatmospheres in the surface of Ganymede. Astrophys. J. 480, L79-L82 (1997).

14. Taube, H. Photochemcial reactions of ozone in solution. Trans. Faraday Soc. 53, 656-665 (1956).

15. Vaida, V., Donaldson, D. J., Strickler, S. J., Stephens, S. L. \& Birks, J. W. A reinvestigation of the electronic spectra of ozone: Condensed-phase effects. J. Phys. Chem. 93, 506-508 (1989).

16. Khare, B. N. et al. Production of optical constants of ice tholin from charged particle irradiation of (1:6) $\mathrm{C}_{2} \mathrm{H}_{6} / \mathrm{H}_{2} \mathrm{O}$ at $77 \mathrm{~K}$. Icarus 103, 290-300 (1993).

17. Thompson, W. R., Murray, B., Khare, B. N. \& Sagan, C. Coloration and darkening of methane clathrate and other ices by charged particle irradiation: Application to the outer solar system. $J$. Geophys. Res. 92, 14933-14947 (1987).

18. Roush, T. L., Cruikshank, D. P. \& Owen, T. C. in Volatiles in the Earth and Solar System (ed Farley, K. A.) 143-153 (Am. Inst. Phys., New York, 1995).

19. Buratti, B. Ganymede and Callisto: Surface textural dichotomies and photometric analysis. Icarus $\mathbf{9 2}$ 312-323 (1991).

20. Buratti, B., Mosher, J. A. \& Johnson, T. V. Albedo and color maps of the saturnian satellites. Icarus 87 339-357 (1990)

21. Lane, A. L., Nelson, R. M. \& Matson, D. L. Evidence for sulphur implantation in Europa's UV absorption band. Nature 292, 38-39 (1981)

22. Noll, K. S., Weaver, H. A. \& Gonnella, A. M. The albedo spectrum of Europa from $2200 \AA$ to $3300 \AA$. $J$. Geophys. Res. 100, 19057-19059 (1995).

23. Noll, K. S., Johnson, R. E., McGrath, M. \& Caldwell, J. J. Detection of $\mathrm{SO}_{2}$ on Callisto with the Hubble Space Telescope. Geophys. Res. Lett. 24, 1139-1142 (1997).

24. Pospieszalska, M. K. \& Johnson, R. E. Magnetospheric ion bombardment profiles of satellites: Europa and Dione. Icarus 78, 1-13 (1989).

25. Tielens, A. G. G. M. \& Hagen, W. Model calculations of the molecular composition of interstellar grain mantles. Astron. Astrophys. 114, 245-260 (1982).

26. Burke, B. F. Detection of planetary systems and the search for evidence of life. Nature 322, 340-341 (1986).

27. Owen, T., Bar-Nun, A. \& Kleinfeld, I. Possible cometary origin of heavy noble gases in the atmospheres of Venus, Earth and Mars. Nature 358, 43-46 (1992).
28. Léger, A., Pirre, M. \& Marceau, F. J. Relevance of oxygen and ozone detections in the search for primitive life in extrasolar planets. Adv. Space Res. 14, 117-122 (1994).

29. Vilas, F., Larson, S. M., Stockstill, K. R. \& Gaffey, M. J. Unraveling the zebra: Clues to the Iapetus dark material composition. Icarus 124, 262-267 (1996).

Acknowledgements. We thank M. J. Bartholomew for sharing her models of visible and IR satellite spectra on which our models of the UV spectrum are based. This work was supported by the Space Telescope Science Institute, which is operated by the Association of Universities for Research in Astronomy, Inc., under NASA contract.

Correspondence should be addressed to K.S.N

\section{Modelling the evolution of human trail systems}

\section{Dirk Helbing ${ }^{\star}$, Joachim Keltsch $\uparrow$ \& Péter Molnár $\ddagger$}

* Institute of Theoretical Physics, University of Stuttgart, Pfaffenwaldring 57/III, 70550 Stuttgart, Germany

$\dagger$ Science-Computing, Hagellocher Weg 71, 72070 Tübingen, Germany

$\ddagger$ Center for Theoretical Studies of Physical Systems, Clark Atlanta University,

James P. Brawley Drive, Atlanta, Georgia 30314, USA

Many human social phenomena, such as $\operatorname{cooperation}^{1-3}$, the growth of settlements ${ }^{4}$, traffic dynamics ${ }^{5-7}$ and pedestrian movement $^{7-10}$, appear to be accessible to mathematical descriptions that invoke self-organization ${ }^{11,12}$. Here we develop a model of pedestrian motion to explore the evolution of trails in urban green spaces such as parks. Our aim is to address such questions as what the topological structures of these trail systems are ${ }^{13}$, and whether optimal path systems can be predicted for urban planning. We use an 'active walker' model ${ }^{14-19}$ that takes into account pedestrian motion and orientation and the concomitant feedbacks with the surrounding environment. Such models have previously been applied to the study of complex structure formation in physi$\mathrm{cal}^{14-16}$, chemical ${ }^{17}$ and biological ${ }^{18,19}$ systems. We find that our model is able to reproduce many of the observed large-scale spatial features of trail systems.

Previous studies have shown that various observed self-organization phenomena in pedestrian crowds can be simulated very realistically. These phenomena include the emergence of lanes of uniform walking direction and oscillatory changes of the passing direction at bottlenecks ${ }^{7,10}$. Another interesting collective effect of pedestrian motion, which we have investigated very recently, is the formation of trail systems in green areas. In many cases, the

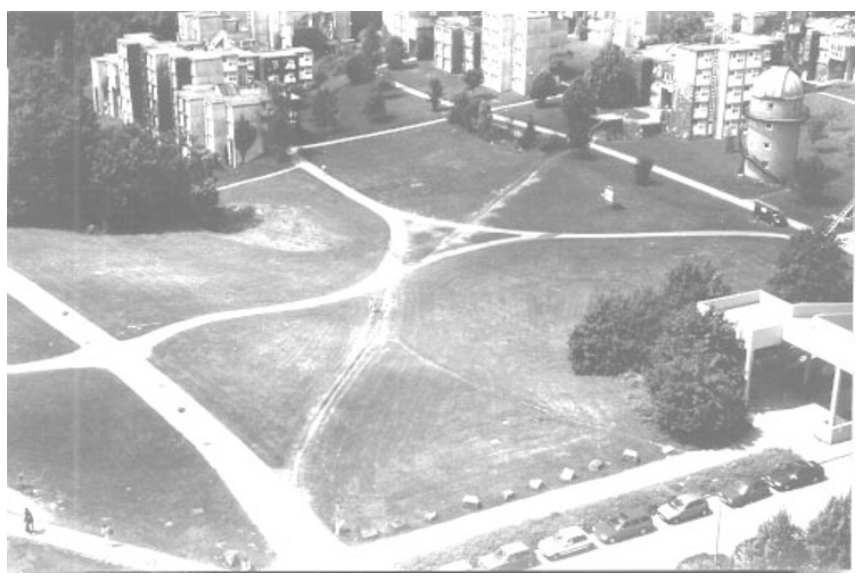

Figure 1 Between the straight, paved ways on the university campus in StuttgartVaihingen a trail system has evolved (centre of the picture). Two types of nodes are observed: intersections of two trails running in a straight line and junctions of two trails which smoothly merge into one trail. 

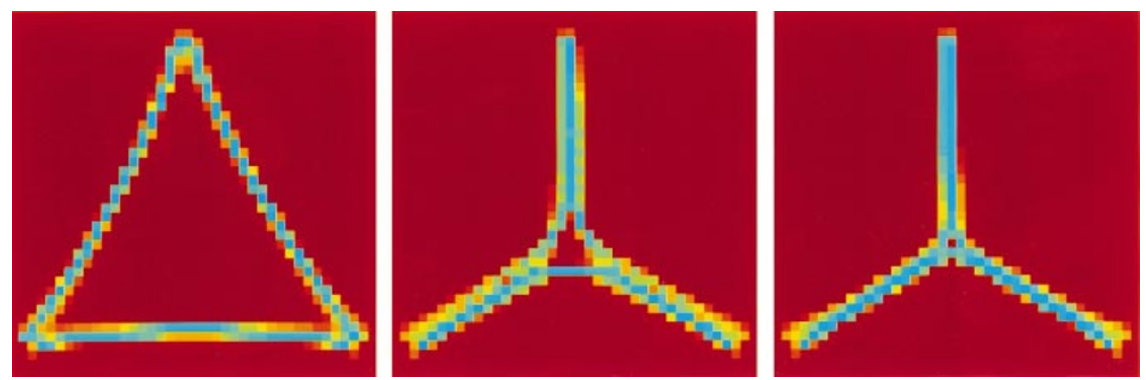

Figure 2 The structure of the emerging trail system (yellow to blue) depends essentially on the attractiveness parameter $\kappa$. If $\kappa$ is small, a direct way system develops (left); if $\kappa$ is large, a minimal way system is formed (right); otherwise, a compromise between both extremes will result (middle) which looks similar to the trail system in the centre of Fig. 1.

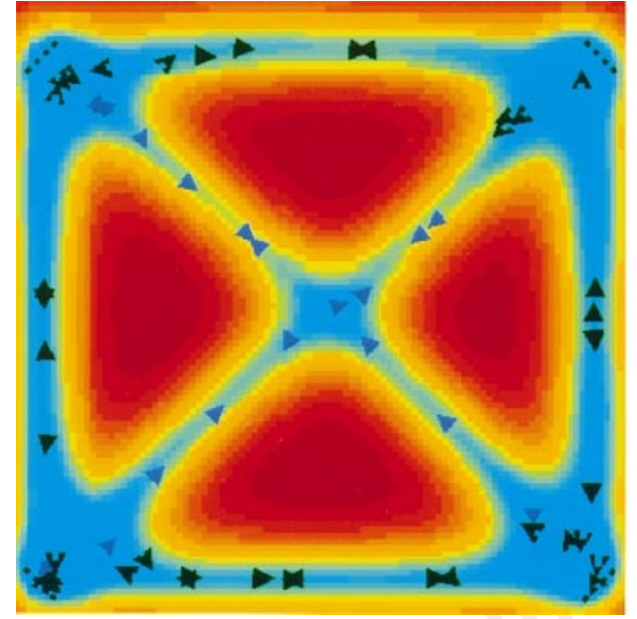

Figure 3 The places and walking directions of pedestrians are represented here by arrows. The trail potential $V_{\mathrm{tr}}(\mathbf{r}, t)$ is represented by a colour scale (red indicates small values, blue indicates large values). Starting with a plain ground, the structure of the trail system changes considerably during the simulation. Initially, pedestrians use the direct ways (left). Because frequently used trails become

pedestrians' desire to take the shortest way and the specific properties of the terrain are insufficient for an explanation of the trail characteristics. It is essential to include the effect of human orientation. To simulate the typical features of trail systems, we have extended the earlier model of pedestrian motion to an activewalker model by introducing equations for environmental changes and their effect on the chosen walking direction.

First, we represent the ground structure at place $\mathbf{r}$ and time $t$ by a function $G(\mathbf{r}, t)$ which reflects the comfort of walking. Trails are characterized by particularly large values of $G$. On the one hand, at their positions $\mathbf{r}=\mathbf{r}_{\alpha}(t)$, all pedestrians $\alpha$ leave footprints on the ground (for example, by trampling down some vegetation). Their intensity is assumed to be $I(\mathbf{r})\left[1-G(\mathbf{r}, t) / G_{\max }(\mathbf{r})\right]$, as the clarity of a trail is limited to a maximum value $G_{\max }(\mathbf{r})$. This causes a saturation effect $\left[1-G(\mathbf{r}, t) / G_{\max }(\mathbf{r})\right]$ of the ground's alteration by new footprints. On the other hand, the ground structure changes owing to the regeneration of vegetation. This will lead to a restoration of the natural ground conditions $G_{0}(\mathbf{r})$ with a certain weathering rate $1 / T(\mathbf{r})$ which is related to the durability $T(\mathbf{r})$ of trails. Thus, the equation of environmental changes reads:

$$
\begin{aligned}
\frac{\mathrm{d} G(\mathbf{r}, t)}{\mathrm{d} t}= & \frac{1}{T(\mathbf{r})}\left[G_{0}(\mathbf{r})-G(\mathbf{r}, t)\right] \\
& +I(\mathbf{r})\left[1-\frac{G(\mathbf{r}, t)}{G_{\max }(\mathbf{r})}\right] \sum_{\alpha} \delta\left(\mathbf{r}-\mathbf{r}_{\alpha}(t)\right)
\end{aligned}
$$

where $\delta\left(\mathbf{r}-\mathbf{r}_{\alpha}\right)$ denotes Dirac's delta function (which yields only a contribution for $\mathbf{r}=\mathbf{r}_{\alpha}$ ).

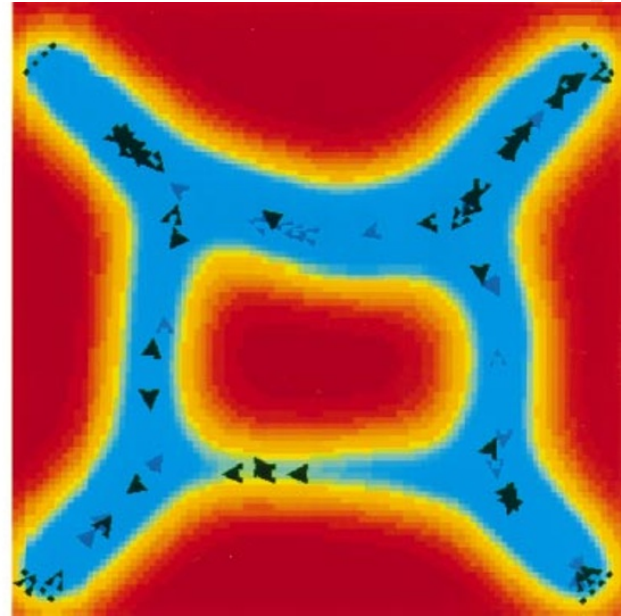

more comfortable, a bundling of trails sets in which reduces the overall length of the trail system (right). The resulting way system (whose asymmetry is caused by differences in the frequency of trail usage) could serve as a planning guideline. It provides a suitable compromise between minimal construction costs and maximal comfort. Moreover, it balances the relative detours of all walkers.

The attractiveness of a trail segment at place $\mathbf{r}$ from the perspective of place $\mathbf{r}_{\alpha}$ decreases with its distance $\left|\mathbf{r}-\mathbf{r}_{\alpha}(t)\right|$ and depends on the visibility $\sigma\left(\mathbf{r}_{\alpha}\right)$. Taking this into account by using a factor $\exp \left(-\left|\mathbf{r}-\mathbf{r}_{\alpha}\right| / \sigma\left(\mathbf{r}_{\alpha}\right)\right)$ and taking the spatial average by integration of the weighted ground structure over the green area, we obtain:

$$
V_{\mathrm{tr}}\left(\mathbf{r}_{\alpha}, t\right)=\int \mathrm{d}^{2} r \mathrm{e}^{-\mid \mathbf{r}-\mathbf{r}_{\alpha} / \sigma\left(\mathbf{r}_{\alpha}\right)} G(\mathbf{r}, t)
$$

The trail potential $V_{\operatorname{tr}}\left(\mathbf{r}_{\alpha}, t\right)$ reflects the attractiveness of walking at place $\mathbf{r}_{\alpha}$. It describes indirect long-range interactions via environmental changes, which are essential for the characteristics of the evolving patterns ${ }^{18}$.

On a plain, homogenous ground, the walking direction $\mathbf{e}_{\alpha}$ of pedestrian $\alpha$ is determined by the direction of the next destination $\mathbf{d}_{\alpha}$, that is $\mathbf{e}_{\alpha}\left(\mathbf{r}_{\alpha}\right)=\left(\mathbf{d}_{\alpha}-\mathbf{r}_{\alpha}\right) /\left|\mathbf{d}_{\alpha}-\mathbf{r}_{\alpha}\right|$. Without a destination, a pedestrian is expected to move into the direction of the largest increase of ground attraction, which is given by the (normalized) gradient $\nabla_{\mathbf{r} \alpha} V_{\mathrm{tr}}\left(\mathbf{r}_{\alpha}, t\right)$ of the trail potential. However, because the choice of the walking direction $\mathbf{e}_{\alpha}$ is influenced by the destination and existing trails at the same time, the orientation relation

$$
\mathbf{e}_{\alpha}\left(\mathbf{r}_{\alpha}, t\right)=\frac{\mathbf{d}_{\alpha}-\mathbf{r}_{\alpha}+\nabla_{\mathrm{r} \alpha} V_{\mathrm{tr}}\left(\mathbf{r}_{\alpha}, t\right)}{\left|\mathbf{d}_{\alpha}-\mathbf{r}_{\alpha}+\nabla_{\mathrm{r} \alpha} V_{\mathrm{tr}}\left(\mathbf{r}_{\alpha}, t\right)\right|}
$$

was taken as the arithmetic average of both effects. Considering cases of rare interactions, the approximate equation of motion of a 

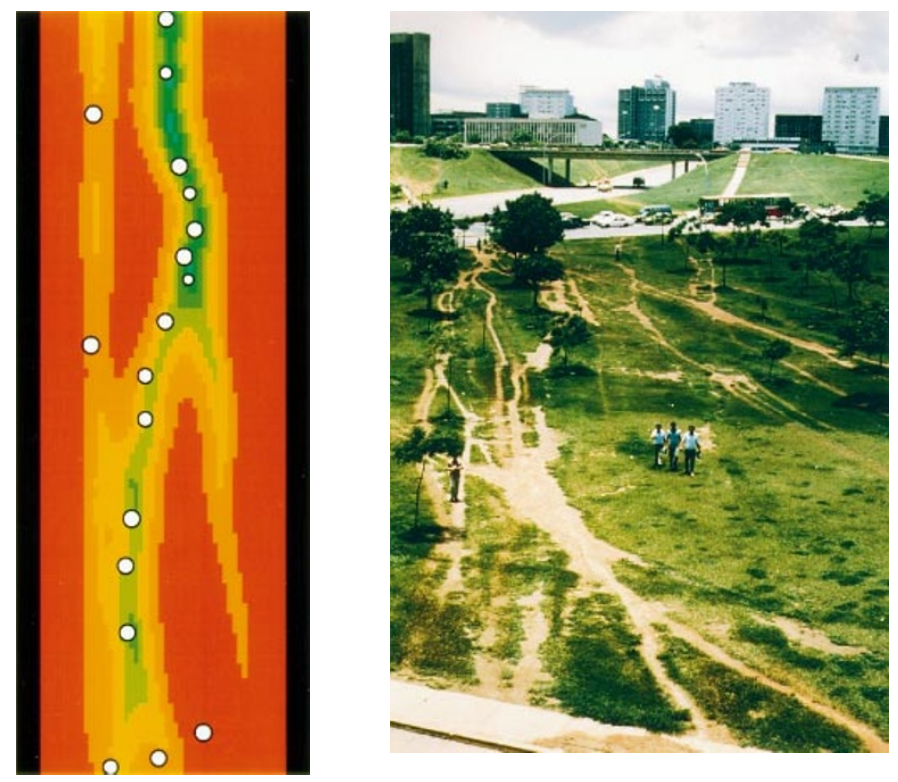

pedestrian $\alpha$ with desired velocity $v_{\alpha}^{0}$ is:

$$
\frac{\mathrm{d} \mathbf{r}_{\alpha}}{\mathrm{d} t}=v_{\alpha}^{0} \mathbf{e}_{\alpha}\left(\mathbf{r}_{\alpha}, t\right)
$$

A comparison of simulation results with photographs shows that the above described model is in good agreement with empirical observations. In particular, the evolution of the unexpected 'island' in the middle of the trail system in Fig. 1 can be correctly described (Fig. 2). The goodness of fit of the model is quite surprising, as it contains only two independent parameters $\kappa=I T / \sigma$ and $\lambda=V^{0} T / \sigma$, where $V^{0}$ denotes the average of the desired velocities $v_{\alpha}^{0}$. This can be shown by scaling the model to dimensionless equations. The parameter $\lambda$ was kept constant.

Our simulations are based on a discretization of the considered area in small quadratic elements of equal size, which converts the integral in equation (2) into a sum. Temporal and spatial derivatives are approximated by difference quotients. The examples we present here begin with plain, homogenous ground. All pedestrians have their own destinations and entry points, from which they start at a randomly chosen point in time. In Fig. 2 (Fig. 3) pedestrians move between all possible pairs of three (four) fixed places; in Fig. 4, the entry points and destinations are distributed over the small ends of the ground.

At the beginning, pedestrians take the direct ways to their respective destinations. But after some time they begin to use already existing trails, as this is more comfortable than clearing new ways. By this, a kind of selection process ${ }^{16,20,21}$ between trails sets in: frequently used trails are more attractive than others. For this reason they are chosen very often, and the resulting reinforcement makes them even more attractive. However, the weathering effect destroys rarely used trails and limits the maximum length of the way system which can be supported by a certain rate of trail usage. As a consequence, the trails begin to bundle, especially where different trails meet or intersect. This explains why pedestrians with different destinations use and produce common parts of the trail system (Figs 2 and 3).

A direct way system (which provides the shortest connections, but covers a lot of space) only develops if all ways are almost equally comfortable. If the advantage $\kappa$ of using existing trails is large, the final trail system is a minimal way system (which is the shortest way system that connects all entry points and destinations). For realistic values of $\kappa$, the evolution of the trail system stops before this state is reached (Fig. 2). Thus, $\kappa$ is related to the average relative detour of the walkers. We conjecture (but cannot yet prove) that the resulting
Figure 4 When pedestrians leave footprints on the ground, trails will develop, and only parts of the ground are used for walking (in contrast to paved areas). The similarity between the simulation result (left) and the trail system on the university campus of Brasilia (right, reproduction by permission of $\mathrm{K}$. Humpert) is obvious.

way system is the shortest one which is compatible with a certain accepted relative detour. In this sense, it yields an optimal compromise between convenience and shortness.

Therefore, we suggest the above model could be used by urban planners and landscape gardeners, who face the task of constructing the most comfortable and convenient way systems at minimal construction costs. For planning purposes, one needs to know the entry points and destinations within the considered area and the rates of usage of their connections. If necessary, these can be estimated by trip chaining models ${ }^{22}$, which are also needed in cases of complex lines of access and sight. The effects of the physical terrain and already existing ways can be taken into account by the function $G_{0}(\mathbf{r})$. By varying the model parameter $\kappa$, the overall length of the resulting trail system can be influenced (Figs 2 and 3). In the same way, one can check its structural stability. We are at present evaluating typical parameter values of $\kappa$ and $\lambda$ by comparison of simulation results with real pedestrian flows which are reconstructed from video films by image processing. These values could be used for designing convenient way systems in residential areas, parks and recreation areas by means of computer simulations (Fig. 3).

It would be interesting to relate our model to work on space syntax ${ }^{23}$. Repulsive interactions between pedestrians, which are relevant in cases of frequent pedestrian interactions, can be taken into account by generalizing equation (4) in accordance with the social force model of pedestrian motion ${ }^{7,10}$; in general, they lead to broader trails.

Received 31 January; accepted 6 May 1997.

1. Axelrod, R. \& Dion, D. The further evolution of cooperation. Science 242, 1385-1390 (1988).

2. Clearwater, S. H., Huberman, B. A. \& Hogg, T. Cooperative solution of constraint satisfaction problems. Science 254, 1181-1183 (1991).

3. Helbing, D. Quantitative Sociodynamics Stochastic Methods and Models of Social Interaction Processes (Kluwer Academic, Dordrecht, 1995).

4. Makse, H. A., Havlin, S. \& Stanley, H. E. Modelling urban growth patterns. Nature 377, 608-612 (1995).

5. Herman, R., Lam, T. \& Prigogine, I. Multilane vehicular traffic and adaptive human behavior. Science 179, 918-920 (1973).

6. Herman, R. \& Prigogine, I. A two-fluid approach to town traffic. Science 204, 148-151 (1979).

7. Helbing, D. Verkehrsdynamik. Neue physikalische Modellierungskonzepte (Springer, Berlin, 1997).

8. Henderson, L. F. The statistics of crowd fluids. Nature 229, 381-383 (1971).

9. Henderson, L. F. Sexual differences in human crowd motion. Nature 240, 353-355 (1972).

10. Helbing, D. \& Molnár, P. Social force model for pedestrian dynamics. Phys. Rev. E 51, 4282-4286 (1995)

11. Haken, H. Advanced Synergetics 2nd edn (Springer, Berlin, 1987).

12. Nicolis, G. \& Prigogine, I. Self-Organization in Nonequilibrium Systems. From Dissipative Structures to Order through Fluctuations (Wiley, New York, 1977)

13. Schenk, M. Untersuchungen zum Fußgängerverhalten Thesis, Univ. Stuttgart (1995).

14. Kayser, D. R., Aberle, L. K., Pochy, R. D. \& Lam, L. Active walker models: tracks and landscapes. Physica A 191, 17-24 (1992).

15. Lam, L. Active walker models for complex systems. Chaos Solitons Fractals 6, 267-285 (1995).

16. Schweitzer, F. \& Schimansky-Geier, L. Clustering of "active" walkers in a two-component system. Physica A 206, 359-379 (1994). 
17. Schimansky-Geier, L., Mieth, M., Rosé, H. \& Malchow, H. Structure formation by active Brownian particles. Phys. Lett. A 207, 140-146 (1995).

18. Ben-Jacob, E. et al. Generic modelling of cooperative growth patterns in bacterial colonies. Nature 368, 46-49 (1994).

19. Stevens, A. \& Schweitzer, F. in Mechanisms of Cell and Tissue Motion (eds Alt, W., Deutsch, A. \& Dunn, G.) 183-192 (Birkhäuser, Basel, 1997).

20. Eigen, M. \& Schuster, P. The Hypercycle (Springer, Berlin, 1979).

21. Feistel, R. \& Ebeling, W. Evolution of Complex Systems. Self-Organization, Entropy and Development (Kluwer, Dordrecht, 1989).

22. Timmermans, H., van der Hagen, X. \& Borgers, A. Transportation systems, retail environments and pedestrian trip chaining behaviour: Modelling issues and applications. Transportation Res. B 26, $45-$ 59 (1992).

23. Hillier, B. Space is a Machine: A Configurational Theory of Architecture (Cambridge Univ. Press, 1996).

Acknowledgements. We thank F. Schweitzer for discussions, and W. Weidlich and M. Treiber for reviews of the manuscript.

Correspondence should be addressed to D.H. (e-mail: helbing@theo2.physik.uni-stuttgart.de).

\section{Current switching of resistive states in magnetoresistive manganites}

\section{A. Asamitsu ${ }^{\star}$, Y. Tomioka ${ }^{\star}$, H. Kuwahara ${ }^{\star} \&$ Y. Tokura ${ }^{\star} \dagger$}

* Joint Research Center for Atom Technology (JRCAT), Tsukuba 305, Japan

$\dagger$ Department of Applied Physics, University of Tokyo, Tokyo 113, Japan

Magnetoresistive devices (based on, for example, magnetic multilayers $^{1}$ ) exhibit large changes in electrical resistance in response to a magnetic field, which has led to dramatic improvements in the data density and reading speed of magnetic recording systems. Manganese oxides having a perovskite structure (the socalled manganites) can exhibit a magnetoresistive response that is many orders of magnitude larger than that found for other materials, and there is therefore hope that these compounds might similarly be exploited for recording applications ${ }^{2-11}$. Here we show that the switching of resistive states in the manganites can be achieved not only by a magnetic field, but also by an electric field. For manganites of the form $\operatorname{Pr}_{1-x} \mathrm{Ca}_{x} \mathrm{MnO}_{3}$, we find that an electrical current (and by implication a static electric field) triggers the collapse of the low-temperature, electrically insulating charge-ordered state to a metallic ferromagnetic state. We suggest that such a phenomenon could be exploited to pattern conducting ferromagnetic domains within an insulating antiferromagnetic matrix, and so provide a route for fabricating micrometre- or nanometre-scale electromagnets.

The carrier-doped manganites with perovskite structure, $\mathrm{R}_{1-x} \mathrm{~A}_{x} \mathrm{MnO}_{3}$ ( $\mathrm{R}$ and $\mathrm{A}$ being rare-earth and alkaline-earth ions, respectively), are well known as conducting ferromagnets below a Curie temperature $T_{\mathrm{C}}$ (ref. 12). The ferromagnetic interaction, called double-exchange interaction ${ }^{13-15}$, between localized $3 d t_{2 g}$ spins (spin quantum number $S=3 / 2$ ) of $\mathrm{Mn}$ ions is mediated by conduction $3 d e_{g}$ carriers. Thus, most of the perovskite manganites show a ferromagnetic (FM) ground state when the holes are optimally doped by the chemical substitution of the perovskite Asite. Among various manganites, $\operatorname{Pr}_{1-x} \mathrm{Ca}_{x} \mathrm{MnO}_{3}$ is unique, showing insulating behaviour over the whole composition $(x)$ range due to its narrow bandwidth of $e_{g}$-electrons ${ }^{9}$. The ground state of $\mathrm{Pr}_{1-x} \mathrm{Ca}_{x} \mathrm{MnO}_{3}$ with $x=0.3-0.5$ is a charge-ordered (CO) antiferromagnetic insulator ${ }^{16,17}$ : the real-space ordering of $1: 1 \mathrm{Mn}^{3+}$ / $\mathrm{Mn}^{4+}$ ions occurs at $T_{\mathrm{CO}} \approx 230 \mathrm{~K}$ and then local spin moments are antiferromagnetically ordered below a Néel temperature, $T_{\mathrm{N}} \approx 170 \mathrm{~K}$, whereas the ferromagnetic double-exchange interaction between $\mathrm{Mn}$ ions is quenched by such a charge-ordering effect. It has been shown ${ }^{8,9}$, however, that this 'charge crystal' can be easily melted into 'charge liquid', namely a FM state, by applying a magnetic field, accompanying an antiferromagnetic-to-ferromagnetic (metamagnetic) phase transition as well as a drastic decrease of the resistivity by more than ten orders of magnitude. Considering the nature of this concomitant metamagnetic and insulator-tometal transition, we may expect that forcedly moved carriers can revive ferromagnetic interaction and trigger the phase transition without a magnetic field. The presence of large hysteresis or metastable states in the course of the melting transition of the charge-ordered state ${ }^{8,9}$ would also favour such a phase switching.

Crystals of $\mathrm{Pr}_{1-x} \mathrm{Ca}_{x} \mathrm{MnO}_{3}(x=0.3)$ were melt-grown by a floating-zone method as described in detail elsewhere ${ }^{8,9}$. The results of powder X-ray diffraction and electron-probe microanalysis indicated that the crystal is single phase with an orthorhombically distorted $(\mathrm{Pbnm})$ lattice. Figure 1 shows the temperature dependence of resistance, $R(T)$, of $\operatorname{Pr}_{1-x} \mathrm{Ca}_{x} \mathrm{MnO}_{3}(x=0.3)$ in zero field at various applied voltages. The charge-ordered state of the $x=0.3$ crystal occurs at $T_{\mathrm{CO}}=230 \mathrm{~K}$, in a similar superlattice pattern to the case of the $x=0.5$ with the arrangement of $\mathrm{Mn}^{3+}: \mathrm{Mn}^{4+}=1: 1$ (ref. 17), but collapses at a relatively small magnetic field as compared with the $x=0.5 \mathrm{crystal}^{8}$. At higher bias voltages, the measured resistance $R$ tends to decrease remarkably as the temperature $T$ is reduced below a spin-canted antiferromagnetic ordering temperature, $T_{\mathrm{CA}}=110 \mathrm{~K}$. The noisy behaviour observed in the $R(T)$ curve below $40 \mathrm{~K}$ at a bias voltage of $300 \mathrm{~V}$ indicates temporal fluctuations between high-resistivity and low-resistivity states. A bias voltage of $700 \mathrm{~V}, R$ at $5 \mathrm{~K}$ is as low as $6 \times 10^{4} \Omega$, reduced by more than five orders of magnitude from the low-voltage $R$-value.

As an example, the change of $R$ at $20 \mathrm{~K}$ in zero field is shown in

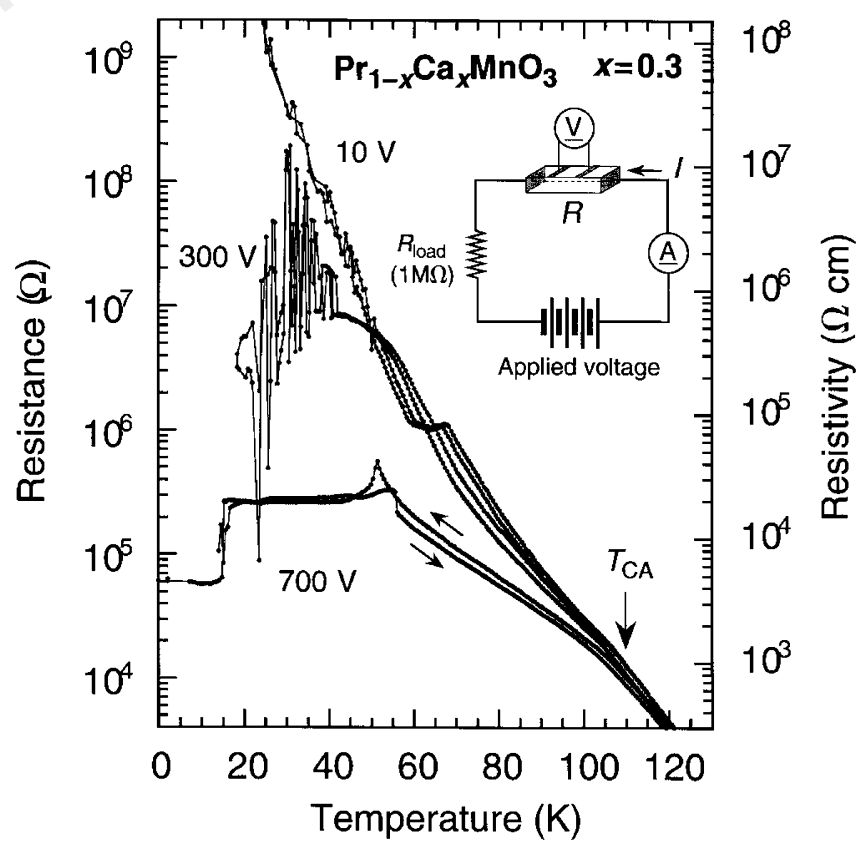

Figure 1 Temperature dependence of resistance of a $\mathrm{Pr}_{1-x} \mathrm{Ca}_{x} \mathrm{MnO}_{3}(x=0.3)$ crystal measured at various applied voltages. The measurements were performed using the circuit shown in the inset. A load resistor of $1 \mathrm{M} \Omega$ was inserted in series to protect the crystal against a burst of current. The crystal was rectangular; $4 \mathrm{~mm}$ long, $1 \mathrm{~mm}$ wide and $0.6 \mathrm{~mm}$ thick. An electrode (for current injection) was made at each end of the crystal (using silver paint) so that the current flow was as uniform as possible. The voltage drop across the crystal was monitored using two other electrodes attached to the surface, which were spaced $\sim 0.9 \mathrm{~mm}$. The resistance was thus measured using the conventional four-wire configuration. The resistivity value on the right ordinate is calculated from current density divided by electric field applied to the crystal, and is meaningful only for the $10 \mathrm{~V}$ (ohmic) case. The spin-canted antiferromagnetic ordering temperature, $T_{C A}$, is $110 \mathrm{~K}$, below which highly nonlinear electric conduction is observed. 